\title{
Nonlinear Analysis of Pull-in Voltage of Twin Micro-Cantilever Beams with Different Dimensions
}

\author{
M. Amin Changizi ${ }^{1}$, Mehrdad Sarafrazi ${ }^{2}$, Ion Stiharu ${ }^{2}$ \\ ${ }^{1}$ Intelliquip Co. \\ 3 W Broad St. Bethlehem, PA, 18031 USA \\ achangizi@intelliquip.com \\ ${ }^{2}$ Concordia University, Department of Mechanical Engineering and Industrial Engineering \\ 1455 De Maisonneuve Blvd. W., Montreal, Quebec, Canada H3G 1M8 \\ m_sarafr@encs.concordia.ca; istih@encs.concordia.ca
}

\begin{abstract}
Micro-cantilevers are micromechanical structures that are being used in detection and sensing as MEMS. The easy usage and the relatively simple fabrication of micro-cantilever beams make them stand as suitable candidates for a wide variety of engineering applications. Dynamic capability of micro-cantilever beams to withstand considerable impacts and even various static loadings make them useful in applications as AFM, inertial sensors, or flow applications. Specific configurations with micro cantilever beams could be used for more advanced applications such as liquid viscosity measurement. The main objective of this research is to investigate the nonlinear dynamic behaviour of two parallel micro cantilever beams with slightly different geometry under the condition of closed to pull-in voltage. The governing differential equations that model closely the dynamical behaviour microcantilever beams under electrostatic field are used in the formulation. The model is based on discreet 2 degree of freedom mass-springdamping. The electrostatic loading induces the dynamical behaviour of two parallel micro cantilever beams expressed in two differential equations that are nonlinear and stiff. A specific algorithm that optimizes the integration steps which is named ISODE is used to perform the computation. The time response and phase diagram to the step-voltage for the mass-spring-damper system is derived numerically and the pull-in voltage of the system is evaluated.
\end{abstract}

Keywords: MEMS, pull-in voltage, twin micro-cantilever beams, nonlinear dynamics

\section{Nomenclature}

$x(t)$ Deflection of the beam

$\dot{\mathrm{x}}$ Velocity

$\ddot{\mathrm{X}}$ Acceleration

c Damping

$\xi$ Damping factor

$\mathrm{m}$ Mass of the beam

$\mathrm{k}$ Spring constant

$\omega_{\mathrm{n}}$ Natural frequency

$\mathrm{f}(\mathrm{t})$ Electrostatic force

$\varepsilon_{0}$ Absolute permittivity of the medium between the beams

\author{
V Applied voltage \\ A Area of cross section on the beam \\ $\mathrm{g}$ Initial distance between the beam and substrate \\ L Length of the beams \\ $b$ Width of the beams \\ $h$ Height of the beams \\ E Young's modulus \\ $v$ Poisson ratio \\ $\rho$ Density
}

\section{Introduction}

During the selection or at the beginning of the design process, the model of the phenomenon of interest is developed. The analysis of the model will point towards the most influential physical quantities, possible instabilities or peculiar behaviour during the operation of the system. This step is fundamental in understanding the sensitivity of the achieved function for the device. Modelling at this stage requires a good understanding of the physics behind the operating principle. It is important to count for all influential phenomena but within the first level of modelling, the most significant effects 
should be counted for. The concern in operating a device is the lack of stability, nonlinearities and catastrophic incidences. Understanding the causes of instabilities and the regions of stable operation largely depend upon the device model. A suitable model will be able to point the designer the critical operating regions and the main cause or reaching those conditions. MEMS devices are among these critical systems which could experience instabilities. The small dimensions as well as the predominance of certain phenomena which appear at small scale in conjunction with unpredictable effects and associated with the lack of perceptibility of the physics at small scale represent main challenges in developing robust models. The pull-in voltage represents one of such critical phenomenon that appears in most of the capacitive based devices. When a difference of potential is applied to an elastically suspended micro-beam, the electrostatic field will induce non-linear electrostatic forces that will attract the components charged with different potentials. When the potential difference increases, the forces will increase due to the potential but also due to the reduced gap between the structures which is produced by the attractive forces. The two effects - larger potential and reduced gap are related and, at a specific point the correlation between the two yields an irreversible attraction between the two structures. This condition is known as the pull-in and the associated potential is known as the pull-in voltage.

According to the formulation yield by equilibrium of forces, it has been derived [1] that pull-in voltage occurs when the equivalent elastic suspended mass reaches the $2 / 3$ of the original gap between the micro-cantilever beam and the substrate because of attraction of the electrodes on the substrate. The operation of micro-cantilever structures under the influence of different types of electric fields has been investigated. Modelling of the phenomenon as well as experimental works were reported $[2,3,4]$. The differential equation that describes the dynamic behaviour of the microsystem consisting of a micro-cantilever beam close to a rigid substrate charged at different potentials can lead to evaluate the pull-in voltage. The governing differential equation of the system can be derived by various common methods such as Hamilton [5] and or one of the energy [6] methods. Due to the high nonlinear trend, several researchers used various methods to linearize and simplify the governing equation.

The pull-in voltage can be calculated numerically by using Finite Element Method (FEM) [7]. Also, the governing equation can be linearized using Taylor series [8]. Runge-Kutta algorithm [9] and the perturbation method [10] represent other-solving methods of the derived equations. The dynamical deflection of the micro-cantilever beam exposed to both harmonic excitations and electric field could also be expressed in a Duffing equation, which represents a preferred formulation as this equation is not stiff. Apart from the above problem-solving methods, there are other approaches that have been considered. For an example the continuum theory for small-scale deflection was scrutinized for micro-cantilever beams and a set of orthogonal functions was established using Taylor series in the governing differential equation [11]. Also, for the small-scale deflection, recent investigations have revealed that the error is commensurate with the length of the beam [4]. The pull in potential remarkably reduces when the structure is dynamically excited at values below the pullin potential values when the structure is excited. Given the large deflection vs. length of the micro-cantilever beams which is characteristic to the small size structures, the previous mentioned solution method is not appropriate [12]. Further, theoretical and experimental investigations demonstrated the effects of changes in width and thickness of the microcantilever beam when the structure is excited close to the resonant frequency.

\section{The Method}

A dynamic model generally accepted for simulation of the vibration of micro-cantilever beams is the discrete lumpedmass system - with one degree of freedom. The equation describing the dynamics of a micro-cantilever beam under the electrostatic field can be expressed as the below equation (1):

$$
\ddot{x}+2 \xi \omega_{n} \dot{x}+\omega_{n}^{2} x=\frac{f(t)}{m}
$$

Where

$$
\ddot{x}=\frac{d^{2} x(t)}{d t^{2}}, \dot{x}=\frac{d x(t)}{d t}, x=x(t)
$$

Figure 1 represents schematics the dynamic model of a non-symmetric twin set of micro-cantilever beams under the electrostatic field by using an equivalent mass-spring-damper system: 


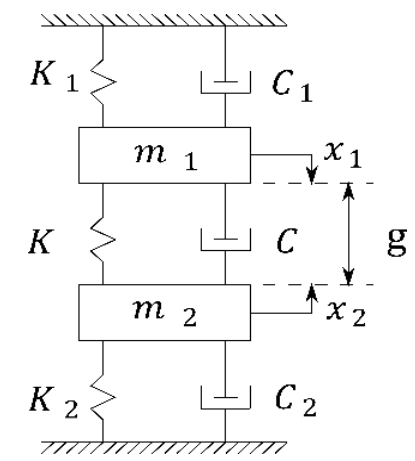

Fig. 1: The schematic view of a mass-spring-damper system of twin micro-cantilever beams with different dimensions.

The above dynamic system can be described as a set of stiff system of nonlinear differential equations:

$$
\left[\begin{array}{cc}
\mathrm{m}_{1} & 0 \\
0 & \mathrm{~m}_{2}
\end{array}\right]\left[\begin{array}{l}
\ddot{\mathrm{x}_{1}}(\mathrm{t}) \\
\ddot{\mathrm{x}_{2}}(\mathrm{t})
\end{array}\right]+\left[\begin{array}{cc}
\mathrm{c}_{1}+\mathrm{c} & -\mathrm{c} \\
-\mathrm{c} & \mathrm{c}_{2}+\mathrm{c}
\end{array}\right]\left[\begin{array}{c}
\dot{\mathrm{x}_{1}}(\mathrm{t}) \\
\dot{\mathrm{x}_{2}}(\mathrm{t})
\end{array}\right]+\left[\begin{array}{cc}
\mathrm{k}_{1}+\mathrm{k} & -\mathrm{k} \\
-\mathrm{k} & \mathrm{k}_{2}+\mathrm{k}
\end{array}\right]\left[\begin{array}{l}
\mathrm{x}_{1}(\mathrm{t}) \\
\mathrm{x}_{2}(\mathrm{t})
\end{array}\right]=\left[\begin{array}{l}
\mathrm{f}_{1}(\mathrm{t}) \\
\mathrm{f}_{2}(\mathrm{t})
\end{array}\right]
$$

The electrostatic effect between the two parallel beams in the micro-structure is notable and it can be considered as an externally applied force. From the energy balance, $f_{1}$ and $f_{2}$ have been found as:

$$
f_{1}=f_{2}=\frac{\varepsilon_{0} A V^{2}}{2\left(g-x_{1}(t)-x_{2}(t)\right)^{2}}
$$

The equation (2) can be expanded into the following equations:

$$
\begin{aligned}
& \mathrm{m}_{1} \frac{\mathrm{d}^{2} \mathrm{x}_{1}(\mathrm{t})}{\mathrm{dt}^{2}}+\left(\mathrm{c}_{1}+\mathrm{c}\right) \frac{\mathrm{dx} \mathrm{x}_{1}(\mathrm{t})}{\mathrm{dt}}+\left(\mathrm{k}_{1}+\mathrm{k}\right) \mathrm{x}_{1}(\mathrm{t})-\mathrm{c} \frac{\mathrm{dx_{2 }}(\mathrm{t})}{\mathrm{dt}}-\mathrm{kx}_{2}(\mathrm{t})=\frac{\varepsilon_{0} \mathrm{~A}_{1} \mathrm{~V}^{2}}{2\left(\mathrm{~g}-\mathrm{x}_{1}(\mathrm{t})-\mathrm{x}_{2}(\mathrm{t})\right)^{2}} \\
& \mathrm{~m}_{2} \frac{\mathrm{d}^{2} \mathrm{x}_{2}(\mathrm{t})}{\mathrm{dt}^{2}}+\left(\mathrm{c}_{2}+\mathrm{c}\right) \frac{\mathrm{dx_{2 }}(\mathrm{t})}{\mathrm{dt}}+\left(\mathrm{k}_{2}+\mathrm{k}\right) \mathrm{x}_{2}(\mathrm{t})-\mathrm{c} \frac{\mathrm{dx_{1 } ( \mathrm { t } )}}{\mathrm{dt}}-\mathrm{kx}_{1}(\mathrm{t})=\frac{\varepsilon_{0} \mathrm{~A}_{2} \mathrm{~V}^{2}}{2\left(\mathrm{~g}-\mathrm{x}_{1}(\mathrm{t})-\mathrm{x}_{2}(\mathrm{t})\right)^{2}}
\end{aligned}
$$

Equation (4) is assigned to the thinner beam (upper beam) and equation (5) is considered for the thicker beam (lower beam). In addition, the initial conditions are defined by the initial deflection and speed at reference position and time for the above ODEs which can be assumed as:

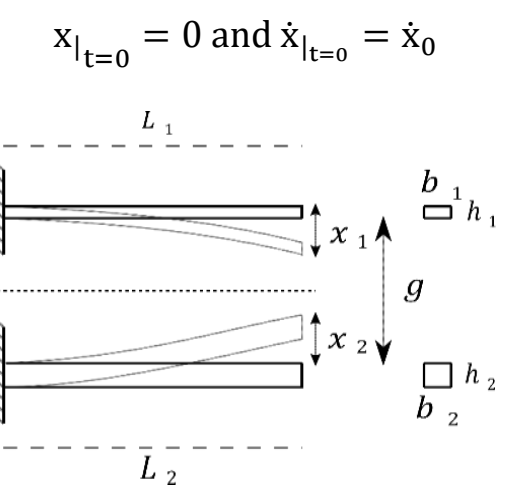

Fig. 2: Non-symmetric twin set micro-cantilever beams (exhibit different dimensions) charged at different potentials. 
Two micro-cantilever beams are parallel and symmetrically positioned. From the above depicted figure, it can be assumed that two beams are same such that $L_{1}=L_{2}, b_{1}=b_{2}$ and $E_{1}=E_{2}$ except the thickness of the beams $h_{1} \neq h_{2}$. Actually, even if certain dimensions are slightly different, the electrostatic attractive force is scaled with regards to the common side of area that are overlapping. After subjecting the beams to electrostatic field, the initial conditions of the beams are still the same as in (6). Figure 3 represents the two micro-cantilever beams under the applied force.

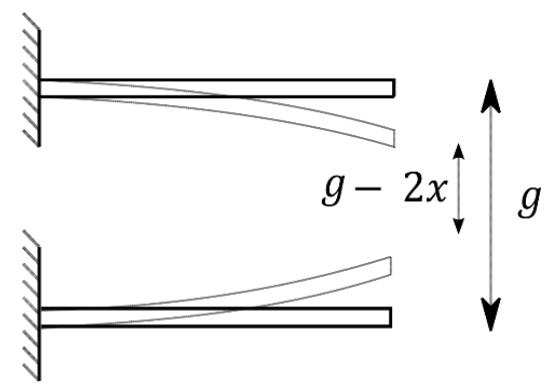

Fig. 2: Two parallel and symmetrical micro-cantilever beams.

The solution of the stiff system of nonlinear differential equation is found using ISODE algorithm. The algorithm makes use of adaptive integration steps such that even when the equations are stiff - close to the pull-in voltage, a correct solution of the problem is yield. The numerical approach was developed for the system of nonlinear ODEs. The results are presented below for the two beams.

In the current work two similar beams were studied under the assumption that the below beam thickness is twice of above beam. The dimensions and properties of beams are as following:

$$
\begin{gathered}
\mathrm{L}_{1}=\mathrm{L}_{2}=200[\mu \mathrm{m}] \\
\mathrm{b}_{1}=\mathrm{b}_{2}=20[\mu \mathrm{m}] \\
\mathrm{h}_{1}=2[\mu \mathrm{m}], \mathrm{h}_{2}=4[\mu \mathrm{m}] \\
\mathrm{E}_{1}=\mathrm{E}_{2}=169[\mathrm{GPa}] \\
\mathrm{g}=10[\mu \mathrm{m}] \\
\xi_{1}=\xi_{2}=0.1 \\
\rho_{1}=\rho_{2}=2300\left[\mathrm{~kg} / \mathrm{m}^{3}\right] \\
v_{1}=v_{2}=0.3 \\
\mathrm{~K}=\frac{2 \mathrm{Ebh}^{2}}{3 \mathrm{~L}^{3}} \\
\omega_{\mathrm{n}}=\sqrt{\frac{\mathrm{K}}{\mathrm{m}}} \\
\mathrm{A}_{1}=\mathrm{A}_{2}(\text { the modified area as in }[13])
\end{gathered}
$$

The numerical solution of the nonlinear system of ODEs in equation 5 will yield to the following results. Figure 4 shows time response of the thinner beam when the voltage is close to the pull-in voltage (145.21 V). Figure 5 shows the phase diagram of the same beam. As one can see, the system reaches to saddle point on the deflection close to 4 [ $\mu \mathrm{m}$ ]. Figure 5 shows the time response of thicker beam. As it is shown in the curve the system goes to one stable condition $(0.3$ $[\mu \mathrm{m}])$ then changes the position and stays on other stable condition $(0.5[\mu \mathrm{m}])$. This phenomenon is illustrated clearly on figure 7 . 


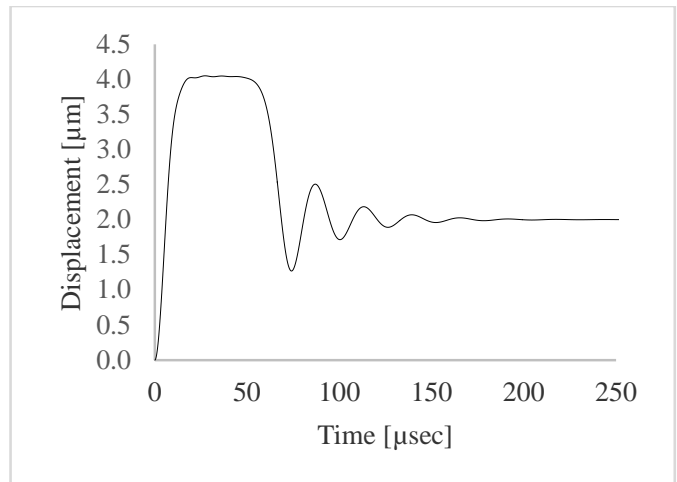

Fig. 4: The deviation of the deflection versus time for the thin beam (upper beam).

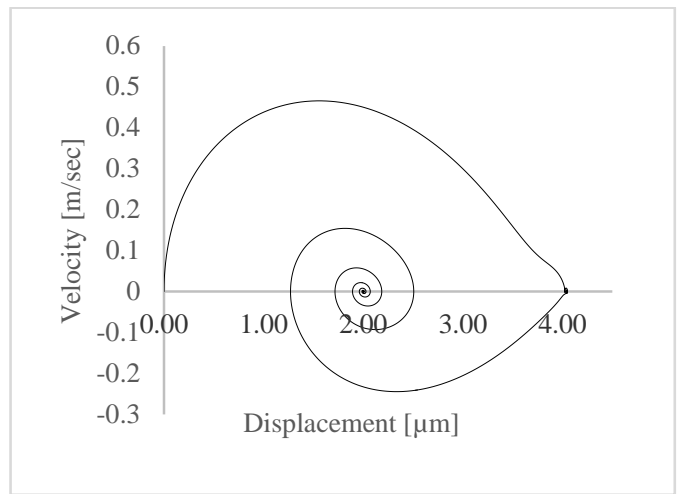

Fig. 5: Phase diagram of the thin beam (upper beam) - changes in velocity versus deflection.

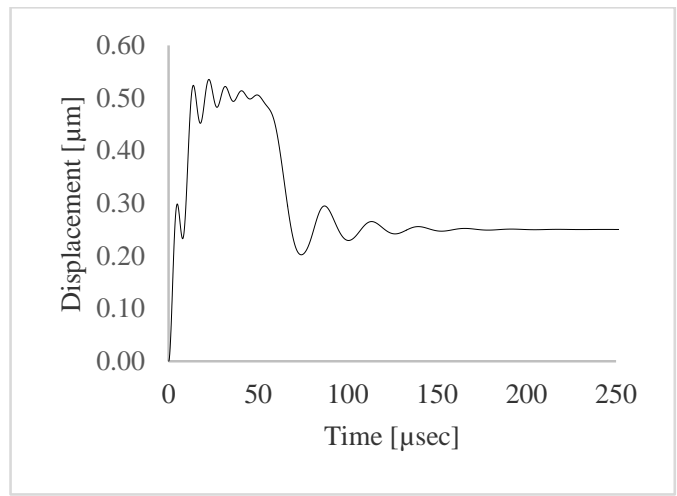

Fig. 6: The deviation of the deflection versus time for the thick beam (lower beam).

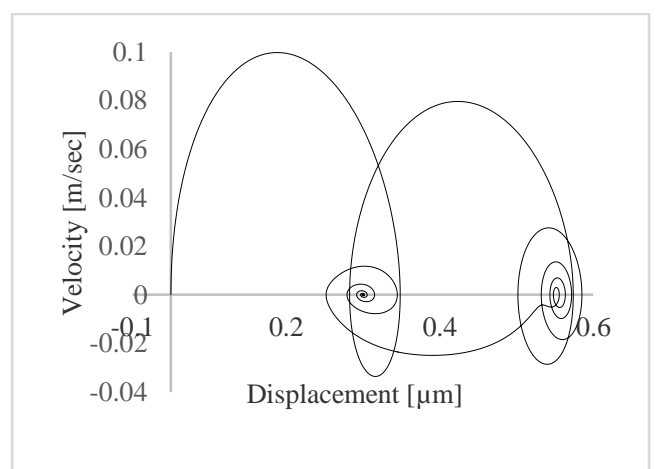

Fig. 7: Phase diagram of the thick beam (lower beam) - unsteady changes in velocity versus deflection. 


\section{Validation}

The validation of the model is carried out using two particular cases with already validated results.

\subsection{The Two Beams are Identical}

In this case, the two beams are considered identical, so thickness were considered same: $h_{1}=h_{2}=2[\mu \mathrm{m}]$. This case was studied previously by authors in [4]. That system was simplified to one nonlinear ODE. The solution of the system of ODEs by assuming two identical beams is illustrated by the time response and the phase portrait in figures 8 and 9 . The results are identical to those in [4]. The plots show the beams response and phase diagram close to pull in voltage.

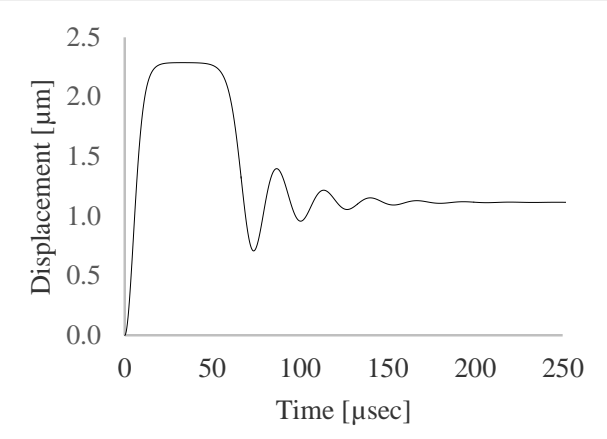

Fig. 8: The deviation of the deflection versus time.

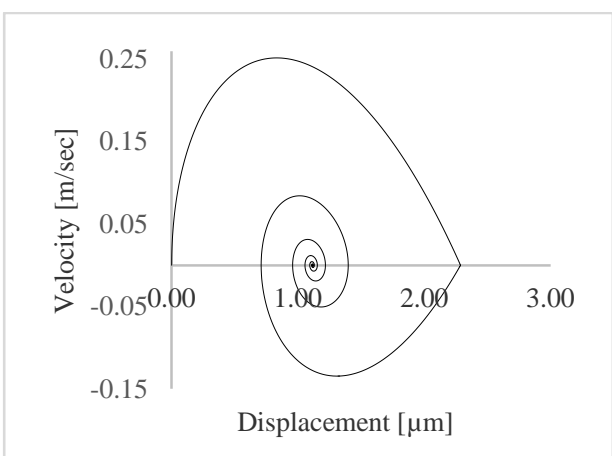

Fig. 9: The deviation of the velocity vs. deflection - phase portrait.

\subsection{Single Beam Pull-in Voltage}

The well-known case of this study is when one of the beams is fixed. This represents the classical problem [1-3]. This case is verified assuming that the bottom beam is extremely thick $(2 \mathrm{~m})$. This makes the bottom beam a substrate. Figure 10 provides the time response of the deflection of the beam. Figure 11 illustrates the phase portrait of the response of the system. The solution as obtained is identical with the results presented in [2] and [12].

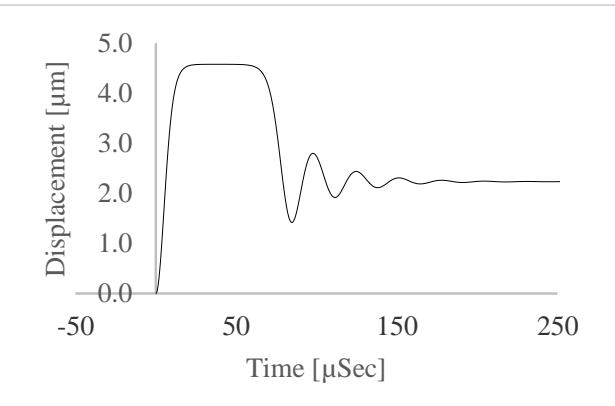

Fig. 10: The deviation of the deflection versus time. 


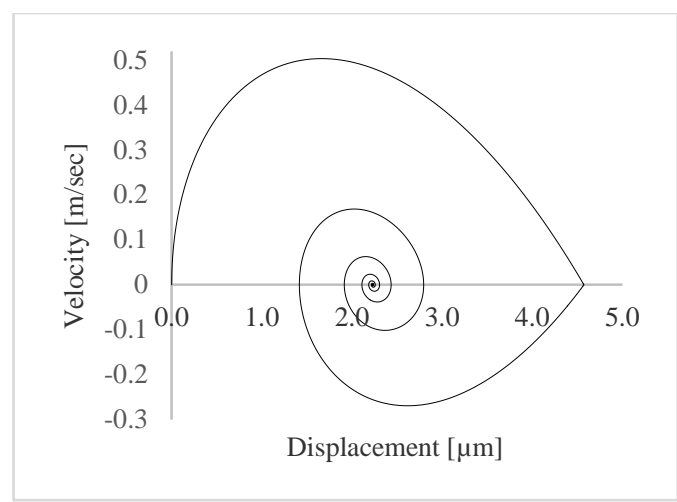

Fig. 11: The deviation of the deflection velocity vs deflection - phase portrait.

\section{Conclusion}

Viscosity is the property of fluids that is difficult to assess. For liquids, disk rheometers are normally used while the minimum sample size is of $100 \mu \mathrm{l}$. The device that is discussed above enables measurement of viscosity through the damping of the oscillation of the two beams which actuate each other through the means of the electrostatic field forces exerted by the difference of potential applied on the two beams. The sample of the analyte could be as low as 1.5 microlitters which represent a significant improvement of the quality of measurement of extremely small specimens. Both pulse measurements and harmonic excitations can enable correlation between the response to excitation and the amplitude of deflection detected as variation in the capacitance. For conductive fluids, the beams could be grown and covered by few tens of nano-meters of $\mathrm{SiO} 2$.

Further investigations are due to the concept of measuring viscosity and density of the fluid as the response of the beams to step or harmonic excitation represents two results that could be diligently investigated.

\section{References}

[1] I. Schiele, J. Huber, B. Hillerich, F. Kozlowski, "Surface-micromachined electrostatic microrelay," Sensors and Actuators A: Physical, vol. 66, no. 1, pp. 345-354, 1998.

[2] M. A. Changizi, I. Stiharu, "A Complete Parametric Study of Pull-In Voltage by Nonlinear Differential Equation," in ASME 2014 International Mechanical Engineering Congress and Exposition (pp. V010T13A026V010T13A026), American Society of Mechanical Engineers.

[3] M. A. Changizi, I. Stiharu, "Lie group analysis of non-linear dynamic of micro structures under electrostatic field," Journal of Computational Methods in Sciences and Engineering, vol. 15, no. 3, pp. 327-338, 2015.

[4] M. A. Changizi, I. Stiharu, "Nonlinear Analysis of Pull-In Voltage of Twin Micro-Cantilever Beams," CDRS 2015, Ottawa, Canada

[5] Y. C. Hu, C. M. Chang, S. C. Huang, "Some design considerations on the electrostatically actuated microstructures," Sensors and Actuators A: Physical, vol. 112, no. 1, pp. 155-161, 2004.

[6] H. Y. Lai, C. C. Liu, "Application of hybrid differential transformation/finite difference method to nonlinear analysis of micro fixed-fixed beam," Microsystem technologies, vol. 15, no. 6, pp. 813-820, 2009.

[7] H. Busta, R. Amantea, D. Furst, J. M. Chen, M. Turowski, C. Mueller, "A MEMS shield structure for controlling pull-in forces and obtaining increased pull-in voltages," Journal of Micromechanics and Microengineering, vol. 11, no. 6 , p. $720,2010$.

[8] M. I. Younis, E. M. Abdel-Rahman, A. Nayfeh, "A reduced-order model for electrically actuated microbeam-based MEMS,” Journal of Microelectromechanical systems, vol. 12, no. 5, pp. 672-680, 2003.

[9] X. Wei, C. Anthony, D. Lowe, M. Ward, "Design and fabrication of a nonlinear micro impact oscillator," Procedia Chemistry, vol. 1, no. 1, pp. 855-858, 2009.

[10] W. Zhang, R. Baskaran, K. L. Turner, "Effect of cubic nonlinearity on auto-parametrically amplified resonant MEMS mass sensor," Sensors and Actuators A: Physical, vol. 102, no. 1, pp. 139-150, 2002.

[11] J. H. Kuang, C. J. Chen, "Dynamic characteristics of shaped micro-actuators solved using the differential quadrature method," Journal of Micromechanics and Microengineering, vol. 14, no. 4, p. 647, 2004. 
[12] M. Amin Changizi, "Geometry and Material Nonlinearity Effects on Static and Dynamics Performance of MEMS," Ph.D. Thesis, Concordia University, Montreal Canada, 2011.

[13] W. Young, R. Budynas, Roark's Formulas for Stress and Strain, 7th Edition, 2011. 